\title{
Complicaciones mecánicas del infarto agudo de miocardio en la era de la COVID
}

\author{
Mechanical complications of acute myocardial infarction in the COVID era \\ Carolina Barajas-Díaz*, Enrique Pérez-de la Sota, María J. López-Gude, Andrea Eixerés-Esteve y \\ José Cortina-Romero \\ Departamento de Cirugía Cardiaca, Hospital Universitario 12 de Octubre, Madrid, España
}

Las complicaciones mecánicas del infarto agudo de miocardio (IAM) tienen una incidencia menor al $1 \%$, debido al aumento en el uso de la reperfusión y particularmente la revascularización percutánea precoz ${ }^{1,2}$. Esto ha mejorado la mortalidad hospitalaria de los pacientes que presentan un IAM, pero no ha demostrado una mejoría significativa en la supervivencia de aquellos que inician con una complicación mecánica, independientemente de si se someten a cirugía o no². Dentro de las complicaciones mecánicas encontramos la rotura subaguda de pared (RSP), la comunicación interventricular (CIV) y la insuficiencia mitral aguda (IMA) secundaria a disfunción o rotura del músculo papilar¹. Presentamos tres casos de complicaciones mecánicas atendidos en nuestro centro en el último mes, asociados a IAM evolucionados y cuyo factor común, recogido en el interrogatorio de ingreso, fue la demora en la consulta a urgencias debida al miedo desencadenado por la pandemia de la enfermedad por coronavirus 2019 (COVID-19).

\section{Caso 1}

Mujer de 70 años sin antecedentes que consulta por disnea súbita asociada a infarto agudo de miocardio con elevación del segmento ST (IAMCEST) anterior evolucionado, con dolor torácico desde 10 días antes. Ingresa en shock cardiogénico, implantándose un balón de contrapulsación intraaórtico (BCIAo) y realizándose coronariografía urgente que evidenció una oclusión de la descendente anterior media y un ecocardiograma que objetivó una CIV apical amplia (Fig. 1) con disfunción ventricular moderada. Se implanta oxigenación por membrana extracorpórea (ECMO) venoarterial para estabilización hemodinámica. Es trasladada a quirófano seis días después, realizándose una exclusión de la CIV con parche de pericardio bovino según técnica de David. Durante el postoperatorio se mantiene en ECMO venoarterial, con mejoría progresiva que permite su retirada a los ocho días desde la cirugía y el BCIAo 24 horas más tarde, coincidiendo con un deterioro hemodinámico brusco, con datos de hipoperfusión y shock, por lo que se sospecha complicación vascular. Se realiza tomografía computarizada abdominopélvica que reporta disección de aorta distal con isquemia en múltiples territorios, falleciendo a las pocas horas.

\section{Caso 2}

Varón de 55 años, fumador, sin otros antecedentes, que consulta por disnea súbita y shock cardiogénico por IAMCEST inferior evolucionado. El paciente presentó dolor torácico opresivo dos semanas previas a su ingreso, sin acudir a valoración. En el ecocardiograma se evidencia dilatación y disfunción biventricular e 


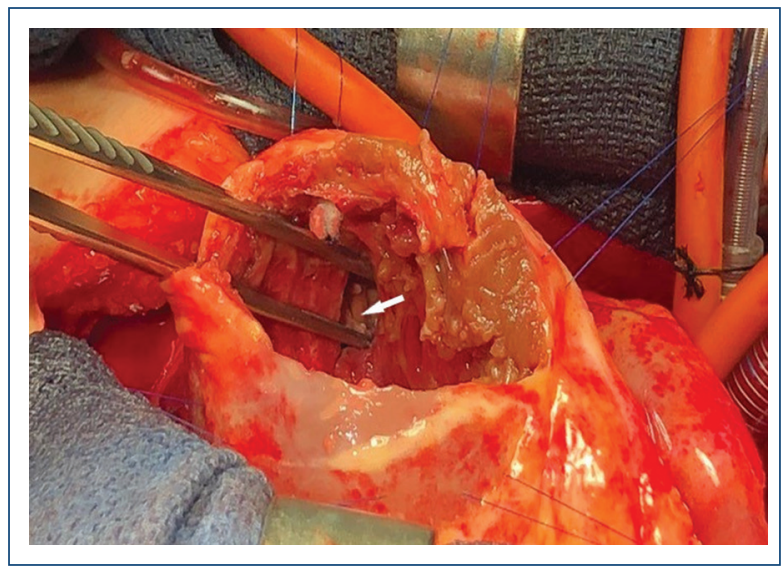

Figura 1. Imagen intraoperatoria de la comunicación interventricular (flecha) y miocardio hemorrágico.

IAM por rotura del músculo papilar posterior. La coronariografía mostró una lesión oclusiva de la coronaria derecha. Se implanta un BCIAo y se traslada a quirófano, realizándose una sustitución valvular mitral con prótesis mecánica. El paciente durante el postoperatorio persiste con disfunción biventricular severa, deterioro hemodinámico, fracaso renal y congestión pulmonar, requiriendo el implante de una ECMO venoarterial a las 24 horas. Tras recuperación de órganos diana, se incluye en lista de trasplante cardiaco, siendo trasplantado a los 11 días desde su ingreso (Fig. 2). Actualmente evoluciona favorablemente.

\section{Caso 3}

Mujer de 69 años hipotiroidea, hipertensa, diabética, dislipémica y fumadora, que presentaba dolor torácico desde hacía tres días con empeoramiento progresivo, por lo que finalmente avisa a emergencias, objetivándose fibrilación auricular de novo e IAMCEST anterior. Se revasculariza percutáneamente la descendente anterior media. En el ecocardiograma se aprecia disfunción ventricular moderada con ventrículo izquierdo ligeramente dilatado y discinesia apical con acinesia de los segmentos distales y medios de la cara anterior, inferoseptal y anteroseptal. Se realiza resonancia magnética cardiaca que informa marcado adelgazamiento e imagen sugestiva de aneurisma apical con trombo intracavitario mural que lo tapiza. Durante el ingreso presentó ictus isquémico hemisférico izquierdo de probable etiología cardioembólica sin secuelas, siendo dada de alta con triple terapia. Sin embargo, la paciente reconsulta siete días más tarde por astenia con incumplimiento terapéutico, deterioro de función

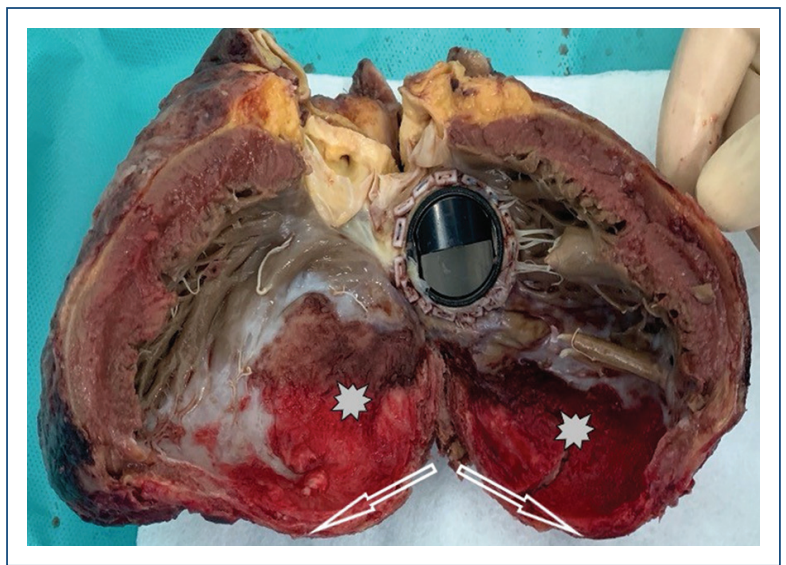

Figura 2. Sección del corazón explantado donde se aprecia la cavidad aneurismática (asteriscos) con adelgazamiento miocárdico secundario al infarto inferior (flechas).

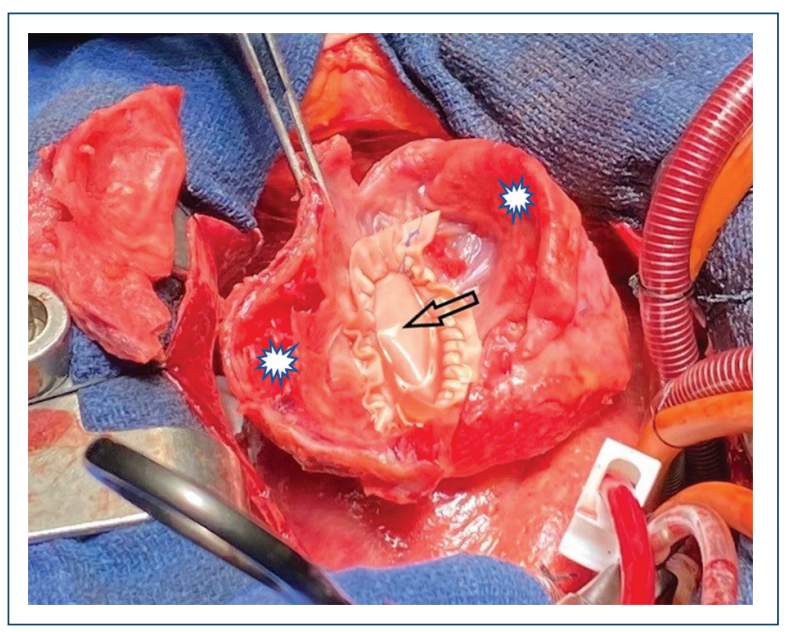

Figura 3. Imagen intraoperatoria del pseudoaneurisma (asteriscos) y exclusión de la zona con parche de pericardio heterólogo (flecha) suturado a miocardio sano.

renal e hipotiroidismo mal controlado, y requiere ingreso hospitalario. Durante su estancia manifiesta disnea súbita y dolor torácico. En el ecocardiograma se objetiva derrame pericárdico severo con datos de taponamiento cardiaco y crecimiento de pseudoaneurisma ventricular, con sospecha de RSP. Se traslada a quirófano, donde se realiza exclusión con parche de pericardio bovino y resección del pseudoaneurisma (Fig. 3), además de implantarse un BCIAo para optimización hemodinámica. La paciente evoluciona favorablemente y es dada de alta a los 12 días tras la intervención. 


\section{Discusión}

En la era de la revascularización percutánea se ha asociado un mayor riesgo de complicaciones mecánicas en el IAM con el retraso en la reperfusión respecto al momento de aparición de los síntomas ${ }^{3}$. Es el caso de nuestros tres pacientes, que retrasaron su consulta a urgencias por el temor a ser ingresados en el hospital y contagiarse de COVID-19, y de similares experiencias comunicadas recientemente ${ }^{4-6}$ que corroboran la importancia de la revascularización percutánea precoz en el infarto con elevación del ST y muestran el impacto negativo que está teniendo sobre ello la actual pandemia por COVID-19.

Como soporte preoperatorio son fundamentales el $\mathrm{BCIAo}$, que ha demostrado disminuir la mortalidad a 30 días en pacientes en shock cardiogénico ${ }^{7}$, y la asistencia ventricular circulatoria tipo ECMO venoarterial, que en la CIV permite retrasar la cirugía en fase aguda; esto mejora la supervivencia ${ }^{8}$, con base en una estabilidad clínica y tejidos más fibróticos debido al proceso de cicatrización tisular, facilitando una reparación quirúrgica más estable.

\section{Financiamiento}

La presente investigación no ha recibido ninguna beca específica de agencias de los sectores público, comercial o sin ánimo de lucro.

\section{Conflicto de intereses}

Los autores declaran no tener ningún conflicto de intereses.

\section{Responsabilidades éticas}

Protección de personas y animales. Los autores declaran que los procedimientos seguidos se conformaron a las normas éticas del comité de experimentación humana responsable y de acuerdo con la Asociación Médica Mundial y la Declaración de Helsinki.

Confidencialidad de los datos. Los autores declaran que han seguido los protocolos de su centro de trabajo sobre la publicación de datos de pacientes.

Derecho a la privacidad y consentimiento informado. Los autores han obtenido el consentimiento informado de los pacientes y/o sujetos referidos en el artículo. Este documento obra en poder del autor de correspondencia.

\section{Bibliografía}

1. Bajaj A, Sethi A, Rathor P, Suppogu N, Sethi A. Acute complications of myocardial infarction in the current era: Diagnosis and management. $J$ Investig Med. 2015;63(7):844-55.

2. Puerto $E$, Viana-Tejedor $A$, Martínez-Sellés $M$, Domínguez-Pérez $L$, Moreno G, Martín-Asenjo R, et al. Temporal trends in mechanical complications of acute myocardial infarction in the elderly. J Am Coll Cardiol. 2018;72(9):959-66.

3. Magalhães $P$, Mateus $P$, Carvalho $S$, Leão S, Cordeiro F, Moreira Jl, et al. Relationship between treatment delay and type of reperfusion therapy and mechanical complications of acute myocardial infarction. Eur Heart J Acute Cardiovasc Care. 2016;5(5):468-74.

4. Alsidawi S, Campbell A, Tamene A, Garcia S. Ventricular septal rupture complicating delayed acute myocardial infarction presentation during the COVID-19 pandemic. JACC Case Rep. 2020;2(10):1595-8.

5. Pilato E, Pinna GB, Parisi V, Manzo R, Comentale G. Mechanical complications of myocardial infarction during COVID-19 pandemic: An Italian single-centre experience. Heart Lung. 2020;49(6):779-82.

6. Atreya AR, Kawamoto K, Yelavarthy P, Arain MA, Cohen DG, Wanamaker BL, et al. Acute myocardial infarction and papillary muscle rupture in the COVID-19 era. JACC Case Rep. 2020;2(10):1637-41.

7. Kettner J, Sramko M, Holek M, Pirk J, Kautzner J. Utility of intra-aortic balloon pump support for ventricular septal rupture and acute mitral regurgitation complicating acute myocardial infarction. Am J Cardiol. 2013;112(11):1709-13.

8. Furui M, Yoshida T, Kakii B, Uchino G, Nishioka H. Strategy of delayed surgery for ventricular septal perforation after acute myocardial infarction. J Cardiol. 2018;71(5):488-93. 